\title{
AN APPROACH FOR DETERMINING THE PERIODICITY OF REGULATION WORK OF RISK TECHNICAL SYSTEMS
}

\section{Nikolay Petrov}

\author{
Prof. Assen Zlatarov University - Bourgas, Bulgaria, nikipetrov@lycos.com
}

\begin{abstract}
The estimation of the regulation work periodicity of the risk technical systems is an extremely important moment for its technical service. Many scientific publications concern this problem but most of them deal with a service process for an infinite technical exploitation period. In the present paper a solution of the problem for a limited interval of technical exploitation is suggested. A reliability model of the observed process is developed as the intensity of the failure flux is chosen for a reliability criteria.
\end{abstract}

Keywords: - Regulation works periodicity, risk technical systems, technical service

\section{INTRODUCTION}

Flux failure intensity $\omega(\Delta t)$ for a final technical exploitation interval $\Delta t$ is estimated on the basis of BDS [1] according $t_{0}$

$$
\omega(\Delta t)=\frac{\sum_{i=1}^{N} r_{i}(\Delta t)}{N_{R T S}(\Delta t) \cdot \sum_{i=1}^{N} \tau_{i}(\Delta t)},
$$

where $N_{R T S}(\Delta t)$ is the number of risk technical systems (RTS) operating system of the same type for the observed interval $\Delta t ; r_{i}(\Delta t)$ - number of failures $i$ of RTS for the observed interval $\Delta t ; \Delta t$ - the observed period of time (1 year for the Bulgarian RTS); $\tau_{i}(\Delta t)$ - life on the $i$ - th RTS for time $\Delta t$.

Flux failure intensity approximation is done through the constant function of time $t$ for observed interval $\Delta t$ represented as a polynomial in formula (2) according to [2]

$$
\omega(\Delta t)=\omega_{0}+a_{1} \cdot t+a_{2} \cdot t^{2}+\ldots+a_{m} \cdot t^{m},
$$

where $a_{1}, a_{2}, \ldots, a_{m}$ are coefficients determined by concrete points in the flux failure intensity function for $t_{i}(i=0,1,2,3, \ldots)$ according $t_{0}[2]$.

The complete equipment statistics RTS and (2) make possible the approximate reliability model of flux failure intensity through a linear function (after an initial moment $t_{0}$ ) in the regulation work interval [3]:

$$
\omega(\Delta t)=\left\{\begin{array}{l}
\omega_{0} \text { for } t=t_{0} ; \\
\omega_{0}+2 V . t \text { for } t>t_{0} .
\end{array}\right.
$$

where $\omega_{0}$ is the interval amount of flux failure intensity of moment $t_{0} ; \quad V=d \omega(\Delta t) / d t$ is the velocity of flux failure intensity increase for the observed interval $\Delta t$.

The model (3) makes possible the optimum regulation work periodicity $\theta_{R W o p t}$, estimation

which provides RTS reliability for the observed period with least regulation works expenses. For that purpose we consider RTS worked out regularly over a certain period of time with periodicity $\theta_{R W}$. During the regulation work the necessary expenses amount to $C_{R W}$ and expenses for current repairs $C_{C R}$.

For the time between the regulation works when the total reconstruction of the RTS is done the leading function of the failure flux $H(\Delta t)$ can be expressed by the following formula according to [1]

$$
H(\Delta t)=\int_{0}^{\theta_{P P}} \omega(\Delta t) \cdot d t .
$$

We consider the expenses for the technical exploitation of the equipment for the period of the regulation work in the folloing two cases

$>E_{C R 1}$ - expenses only for current repairs.

$>E_{C R 2}$ - expenses for regulation works and current repairs. 
Having in mind the above mentioned and $[4,5,6]$ we can calculate savings from the technical exploitations $S_{T E}$ of the RTS

$$
S_{T E}=E_{C R 1}-E_{C R 2}=C_{C R}\left[\begin{array}{l}
H(\Delta t)-m H\left(\theta_{R W}\right) \\
-H\left(k_{R W} \cdot \theta_{R W}\right)
\end{array}\right]-C_{R W} m
$$

where $m$ is the number of regulation work for the observed period of time.

The number of regulation work for the whole period of technical exploitation is defined according to [6] from

$$
m=\frac{t}{\theta_{R W}}-k_{R W},
$$

where $k_{R W}$ is the number of regulation work periods for the time $t$.

In formula (5) member $k_{R W} \cdot \theta_{R W}$ is life in the final interval on the RTS for work under the condition $\left(0<k_{R W} \cdot \theta_{R W}<T_{R T E}\right)$, where $T_{R T E}$ is the technical resource till the end of the technical exploitation.

Equations (4) and (6) are used for (5) investigation if only the current time parameter will be in time limit. We get the following

$$
\begin{aligned}
& S_{E R}=C_{C R}\left[\omega_{0} \Delta t+V(\Delta t)^{2}\right]-\left(\frac{\Delta t}{\theta_{R W}}-k_{R W}\right)\left[C_{C R} \cdot \omega_{0} \cdot \theta_{R W}\right. \\
& \left.+C_{C R} \cdot V \cdot\left(\theta_{R W}-t_{0}\right)^{2}+C_{R W}\right]-C_{C R} \cdot\left[\begin{array}{l}
\omega_{0} \cdot k_{R W} \cdot \theta_{R W}+V . \\
\cdot\left(k_{R W} \cdot \theta_{R W}-t_{0}\right)^{2}
\end{array}\right] .
\end{aligned}
$$

After investigating equation (7) we get the following differential equation

$$
\begin{gathered}
\left(C_{R W}+C_{C R} \cdot V t_{0}^{2}+C_{C R} V \theta_{R W}^{2}\right) k_{R W}^{\prime}-2 C_{C R} V \theta_{R W}^{2} k_{R W} . \\
. k_{R W}^{\prime}-2 C_{C R} V k_{R W}^{2} \theta_{R W}+2 C_{C R} V \theta_{R W} k_{R W}- \\
-C_{C R} V \Delta t+\frac{C_{C R} V . \Delta t \cdot t_{0}^{2}}{\theta_{R W}^{2}}+\frac{C_{R W} \cdot \Delta t}{\theta_{R W}^{2}}=0 .
\end{gathered}
$$

There is no solution to (8) so we do:

$$
u=C_{C R} \cdot V \cdot \theta_{R W}^{2} \cdot k_{R W}^{2}+\left(\begin{array}{c}
C_{R W}+C_{C R} \cdot V \cdot \theta_{R W}^{2}+ \\
+2 C_{C R} \cdot V \cdot t_{0}^{2}
\end{array}\right) \cdot k_{R W} .
$$

Filling (8) with (9) we get the following equation:

$$
u^{\prime}+\frac{C_{R W} \cdot \Delta t}{\theta_{C R}^{2}}+\frac{C_{C R} \cdot V \cdot \Delta t \cdot t_{0}^{2}}{\theta_{R W}^{2}}-C_{C R} \cdot V \cdot \Delta t=0 .
$$

Its solution is as folows:

$$
u=C_{C R} \cdot V \cdot \Delta t \cdot \theta_{R W}+\frac{C_{C R} \cdot V \cdot \Delta t \cdot t_{0}^{2}}{\theta_{R W}}+\frac{C_{R W} \cdot \Delta t}{\theta_{R W}}+C_{i} .
$$

We equalize (9) and (11) and get the algebraic equation

$$
\begin{gathered}
C_{C R} V \theta_{R W}^{3} k_{R W}^{3}-\left(C_{R W} \theta_{R W}+C_{C R} V \theta_{R W}^{3}+C_{C R} V t_{0}^{2} \theta_{R W}\right) . \\
k_{R W}+C_{C R} \cdot V \cdot \Delta t \cdot \theta_{R W}^{2}+C_{C R} \cdot V \cdot \Delta t \cdot t_{0}^{2}+ \\
+C_{R W} \cdot \Delta t+C_{i} \cdot \theta_{R W}=0 .
\end{gathered}
$$

In order to define the integration constant $C_{i}$ we use $\theta_{R W}=0,5 . \Delta t$, from which follows $m=1$, $k_{R W}=1$. We use these equation (12) and we get

$$
C_{i}=-C_{R W}-C_{C R} \cdot V \cdot t_{0}^{2}-\frac{C_{C R} \cdot V \cdot(\Delta t)^{2}}{2} .
$$

Filling equation (12) with $C_{i}$ from (13) we receive the final

$$
\begin{gathered}
C_{C R} \cdot V \cdot \theta_{R W}^{3} \cdot k_{R W}^{2}-\left(C_{R W} \theta_{R W}+C_{C R} V \theta_{R W}^{3}+C_{C R} V t_{0}^{2} \theta_{R W}\right) . \\
. k_{R W}+C_{C R} \cdot V \cdot \Delta t \cdot \theta_{R W}^{2}+C_{C R} \cdot V \cdot \Delta t \cdot t_{0}^{2}+C_{R W} \cdot \Delta t- \\
-\left(C_{R W}+C_{C R} \cdot V \cdot t_{0}^{2}+\frac{C_{C R} \cdot V \cdot(\Delta t)^{2}}{2}\right) \cdot \theta_{R W}=0 .
\end{gathered}
$$

Equation (14) proves the dependence of $k_{R W}$ and $\theta_{R W}$. Filling in it $k_{R W}=\left(\Delta t-m \cdot \theta_{R W}\right) \cdot \theta_{R W}^{-1}$ we get the algebraic equation which is the link between $\theta_{R W}$ and $m$. It is the following

$$
\begin{aligned}
& \left(C_{C R} V m^{2}+C_{C R} V m\right) \cdot \theta_{R W}^{2}-2 C_{C R} \cdot V \cdot \Delta t \cdot m \cdot \theta_{R W}+0,5 C_{C R} \cdot \\
& . V \cdot(\Delta t)^{2}+C_{R W} \cdot m+C_{C R} \cdot V \cdot t_{0}^{2} \cdot m-C_{C R} \cdot V \cdot t_{0}^{2}-C_{R W}=0 .
\end{aligned}
$$

Solving (15) for $\theta_{R W}$ we get

$$
\theta_{R W}=\frac{\Delta t}{m+1}+\sqrt{\begin{array}{c}
\frac{(\Delta t)^{2} \cdot(m-1)}{2 m \cdot(m+1)^{2}}-\frac{C_{R W} \cdot(m-1)}{C_{C R} \cdot V \cdot m(m+1)} \\
-\frac{t_{0}^{2} \cdot(m-1)}{m(m+1)}
\end{array}}
$$

In order to get the real quantities on $\theta_{R W}$ equation (16) is solved under the following terms

$$
1 \leq m \leq \frac{C_{C R} \cdot V \cdot(\Delta t)^{2}-2 C_{R W}-2 C_{C R} \cdot V \cdot t_{0}^{2}}{2 C_{R W}+2 C_{C R} V \cdot t_{0}^{2}} .
$$

Filling (15) with (16) we get

$$
k_{R W}=k_{R W}(m)=\frac{\Delta t-m \sqrt{A}}{\Delta t-(m+1) \sqrt{A}},
$$

where 


$$
A=\frac{(\Delta t)^{2} \cdot(m-1)}{2 m(m+1)^{2}}-\frac{C_{R W}(m-1)}{C_{C R} \cdot V \cdot m(m+1)}-\frac{t_{0}^{2}(m-1)}{m(m+1)} .
$$

\section{CONCLUSIONS}

From equation (16) and (18) we can estimate the regulation works periodicity of the risk technical systems. These equations make possible the estimation of the period numbers with definite regulation works for a certain technical exploitation period.

\section{REFERENCES}

[1]. BDS27.002-86. Reliability in technics. Main therms and determinations. Committee for quality. Sofia, 1987. (in Bulgarian)

[2]. N.Petrov. Optimization and control for technical exploitation on military aviation systems. VA "Rakovski", Sofia, 2002. (in Bulgarian)

[3]. A.Rybalov, V.Gladilin. Method for finding of main characteristics of process of failures recovery in complex aviation systems. VVIA "Zhukovski", Moscow, 1968. (in Russian)

[4]. Ya.Khehagurova. Reliability of automated control systems. "Vysshaya shkola", Moscow, 1979. (in Russian)
[5]. G.Panayotova. Simple waves for certain hyperbolic systems of PDEs", "International Journal of Differential Equations and Applications". Volume 6, No.32002.

[6]. N.Tencheva, I.Kalchev. Evaluation of reliability of DMS with built-in structural reservation. SP. "Technicheska mysl", Vol. 1-2, 2001, pp. 3-10. (in Bulgarian)

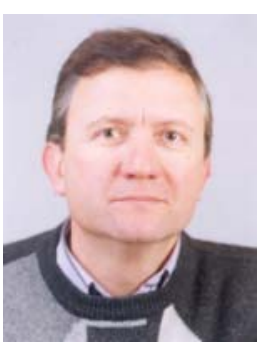

Nickolay Ivanov Petrov was born on 21 December 1953.

Education: 1972-1977 Georgi Benkovski Higher Military Institution of Air Forces, Dolna, Mitropolia. Specialty "Communicative equipping of aircraft".

1990-1995 - Doctor's degree

in Engineering, Military Academy, Institute for Perspective Defence Research, Sofia.

1999-2002 - Dr. Sc. in Engineering (Automated Systems for Information Technology And Management). 